\title{
A NEW CHARACTERIZATION OF TREES
}

\author{
L. E. WARD, JR. \\ (Communicated by Doug Curtis)
}

\begin{abstract}
It is proved that a continuum is a tree if and only if for each pair of nondegenerate subcontinua $K$ and $L$ with $K \subset L$, it follows that $K$ contains a cutpoint of $L$.
\end{abstract}

A connected Hausdorff space $X$ is said to be tree-like [1] if each pair of distinct points can be separated by a third point, i.e., if $x, y \in X$ and $x \neq y$ then there exists $z \in X$ so that $X-\{z\}=A \cup B$ where $A$ and $B$ are disjoint open sets, $x \in A$ and $y \in B$. A tree is a compact tree-like space. It is well known [5] that a continuum is a dendrite if and only if it is a metrizable tree.

It is a theorem of R. L. Moore [3] that a metrizable continuum $X$ is a dendrite if and only if each nondegenerate subcontinuum of $X$ contains uncountably many cutpoints of $X$. In the setting of Hausdorff continua this theorem does not generalize to yield a characterization of trees. (For a counterexample, see [4, Example 9].) Moore's theorem is now sixty years old, and trees have been intensively studied for over forty years and are quite well understood. Therefore, it is surprising that a suitable variation of Moore's theorem, permitting a characterization of trees, has so far eluded discovery. In this note we obtain such a variation.

A point $z$ of a connected space $X$ is a cutpoint of $X$ provided $X-\{z\}$ is not connected. A continuum is a compact connected Hausdorff space. A continuum $X$ is hereditarily unicoherent if, for each pair of subcontinua $A$ and $B$ it follows that $A \cap B$ is connected. An ordered continuum is a nondegenerate continuum with exactly two non-cutpoints. Finally, a continuum $X$ is said to satisfy property $T$ provided, for each pair of nondegenerate subcontinua $K$ and $L$ with $K \subset L$, it follows that $K$ contains a cutpoint of $L$.

THEOREM 1. A continuum is a tree if and only if it satisfies the property $T$.

ProOF. Suppose $X$ is a tree and that $K$ and $L$ are nondegenerate subcontinua with $K \subset L$. Let $x$ and $y$ be elements of $K$. Since $L$ is a tree there exists $z \in L$ such that $L-\{z\}=L_{1} \cup L_{2}$, a separation, with $x \in L_{1}$ and $y \in L_{2}$. But $K$ is a subcontinuum of $L$, so $z \in K$. Hence $K$ contains a cutpoint of $L$.

Now assume $X$ is a continuum satisfying property $T$. The proof that $X$ is a tree will be dividied into 4 parts.

1. $X$ is hereditarily unicoherent. For suppose there are subcontinua $A$ and $B$ of $X$ such that $A \cap B=P \cup Q$ where $P$ and $Q$ are nonempty separated sets. Then

Received by the editors June 12, 1987 and, in revised form, October 9, 1987.

1980 Mathematics Subject Classification (1985 Revision). Primary 54F20, 54F50, 54F65; Secondary $54 \mathrm{~F} 55$.

Key words and phrases. Tree, tree-like, dendrite, cutpoint, continuum, property $T$. 
$\overline{B-A}$ contains a continuum $I$ which is irreducible between $P$ and $Q$. Let $x \in I-A$ and suppose $C$ and $D$ are components of $I-\{x\}$ which meet $P$ and $Q$, respectively. (It is possible that $C=D$.) Then $\bar{C} \cup \bar{D}=I$ by the irreducibility of $I$, and $C \cup D \cup A$ is a connected set which is dense in $A \cup I-\{x\}$. Accordingly, no point of $I-A$ is a cutpoint of $A \cup I$. Therefore, if $I_{1}$ is a nondegenerate subcontinuum of $I$ which is disjoint from $A$, then $I_{1}$ contains no cutpoint of $A \cup I$, contradicting the hypothesis that $X$ satisfies property $T$. Therefore $X$ is hereditarily unicoherent.

2. It follows at once that if $\varnothing \neq S \subset X$ then $X$ contains a unique subcontinuum irreducible about $S$. If $S=\{x, y\}$ we denote that subcontinuum by $[x, y]$. Note that if $t \in[x, y]$ then $[x, y]=[x, t] \cup[t, y]$ by irreducibility. Moreover, if $[x, y]$ is contained in a subcontinuum $L$, then it is clear that $[x, y]-\{x, y\}$ contains a nondegenerate subcontinuum. Therefore, by property $T$, the set $[x, y]-\{x, y\}$ contains a cutpoint of $L$. We will use this fact repeatedly in the rest of the proof.

3. If $x$ and $y$ are distinct points of $X$ then $[x, y]$ is an ordered continuum. It will suffice to show that if $z \in[x, y]-\{x, y\}$ then $z$ is a cutpoint of $[x, y]$. If the contrary obtains then $[x, y]=[x, z] \cup[z, y]$ where $[x, z] \cap[z, y]$ is nondegenerate. Since $X$ is hereditarily unicoherent, $[x, z] \cap[z, y]$ is a continuum and therefore contains a cutpoint $w$ of $[x, y]$, by property $T$. Therefore $[x, y]=[x, w] \cup[w, y]$ with $[x, w] \cap$ $[w, y]=\{w\}$, and we may assume $z \in[x, w]-\{w\}$. Therefore $[x, z] \subset[x, w]$, and since $w \in[x, z]$ it follows that $[x, z]=[x, w]$; however $[w, y]$ is properly contained in $[z, y]$ and so $z \notin[w, y]$. Again by property $T$, the set $[z, w]-\{z, w\}$ contains a cutpoint $w^{\prime}$ of $[x, y]$. Since $w^{\prime} \in[z, w] \subset[x, w]$, it follows that $w \in\left[w^{\prime}, y\right]$. Thus we have

$$
[x, y]=\left[x, w^{\prime}\right] \cup\left[w^{\prime}, w\right] \cup[w, y]
$$

and so $z \in\left[x, w^{\prime}\right] \cup\left[w^{\prime}, w\right]=[x, w]$. Because $[x, z]=[x, w]$ it is clear that $z \notin\left[x, w^{\prime}\right]$ and therefore $z \in\left[w^{\prime}, w\right]$. Applying property $T$ again, the set $\left[w^{\prime}, w\right]-\left\{w^{\prime}, w\right\}$ contains a cutpoint $w^{\prime \prime}$ of $[x, y]$. Clearly $\left[w^{\prime}, w\right]=\left[w^{\prime}, w^{\prime \prime}\right] \cup\left[w^{\prime \prime}, w\right]$ and $\left[w^{\prime}, w^{\prime \prime}\right] \cap$ $\left[w^{\prime \prime}, w\right] \subset\left[x, w^{\prime \prime}\right] \cap\left[w^{\prime \prime}, y\right]=\left\{w^{\prime \prime}\right\}$. Since $z \in\left[w^{\prime}, w\right]$, either $z \in\left[w^{\prime}, w^{\prime \prime}\right]$ or $z \in$ $\left[w^{\prime \prime}, w\right]$. But if $z \in\left[w^{\prime}, w^{\prime \prime}\right]$ then $[x, z] \subset\left[x, w^{\prime \prime}\right] \neq[x, w]$, which is impossible. And if $z \in\left[w^{\prime \prime}, w\right]$ then $[z, w] \subset\left[w^{\prime \prime}, w\right]$ and hence $w^{\prime} \notin[z, w]$, which is also impossible. We have arrived at a contradiction and therefore $z$ is a cutpoint of $[x, y]$.

4. $X$ is a tree. Suppose $x$ and $y$ are distinct elements of $X$ and $z \in[x, y]-\{x, y\}$. We will show that $z$ is a cutpoint of $X$ and that $z$ separates $x$ and $y$ in $X$.

For each $t \in X-\{z\}$ let $A_{t}$ denote the union of all ordered continua which contain $t$ and are contained in $X-\{z\}$. (That is, $A_{t}$ is the Hausdorff space analog of an arc-component of $X-\{z\}$.) Note that $[z, t]-\{z\} \subset A_{t}$. Also, if $s \in A_{t}$ then $A_{s}=A_{t}$, and hence we may regard $t$ as an arbitrary point of $A_{t}$. Suppose there exists a net $t_{\alpha}$ with values in $X-A_{t}$ and $\lim t_{\alpha}=t$. Let $Z=\overline{\bigcup\left\{\left[t_{\alpha}, t\right]\right\}}$; clearly $Z$ is a continuum. Since $t_{\alpha} \notin A_{t}$ it follows that $z \in\left[t_{\alpha}, t\right]$ for each $\alpha$ and hence $z \in Z$. Therefore $[z, t] \subset Z$ so that by property $T,[z, t]-\{z, t\}$ contains a cutpoint $p$ of $Z$. Thus $Z$ is the union of proper subcontinua $Z_{1}$ and $Z_{2}$ with $Z_{1} \cap Z_{2}=\{p\}$. Moreover, because $[p, t] \subset[z, t]-\{z\}$, it is clear that $p \in A_{t}$. If $z \in Z_{1}$ and $t \in Z_{2}$ then eventually $t_{\alpha} \in Z_{2}$ so that $\left[t_{\alpha}, p\right] \subset Z_{2}$. It follows that $t_{\alpha} \in A_{t}$, a contradiction. Therefore we may assume that both $z$ and $t$ lie in $Z_{1}$. If there exists $\alpha_{1}$ such that $t_{\alpha_{1}} \in Z_{2}$ then $p \in\left[t_{\alpha_{1}}, z\right]$. Since $p \in A_{t}$ it follows that $t_{\alpha_{1}} \in A_{t}$, a contradiction. Consequently $t_{\alpha} \in Z_{1}$ for all $\alpha$ and therefore $Z \subset Z_{1}$. But this 
contradicts the assumption that $Z_{1}$ is a proper subcontinuum of $Z$. Therefore, no such net $t_{\alpha}$ can exist and we conclude that each set $A_{t}$ is open. Since $z$ cuts $[x, y]$ it is clear that $A_{x}$ and $A_{y}$ are disjoint. That is to say, $X-\{z\}$ is not connected and $z$ separates $x$ and $y$ in $X$.

I have learned recently that E. Tymchatyn and W. Bula have obtained another proof of Theorem 1.

Let us say that a continuum satisfies property $M$ if each of its nondegenerate subcontinua contains uncountably many cutpoints of $X$. One sees easily that a continuum satisfies property $T$ if and only if each subcontinuum satisfies property $M$. The natural question arises: suppose $P$ is a property of metrizable continua which is equivalent to being a dendrite; among Hausdorff continua when is the condition that each subcontinuum satisfies $P$ equivalent to being a tree? We conclude this note with two more propertiees of this sort.

Let us say that a continuum satisfies property $W$ if each of its points is either a cutpoint or an endpoint. Wilder [6] proved that a metrizable continuum is a dendrite if and only if it satisfies property $W$, but this result does not generalize to Hausdorff continua.

THEOREM 2. A continuum is a tree if and only if each of its nondegenerate subcontinua satisfies property $W$.

PROOF. Every subcontinuum of a tree is a tree and it is known [4] that each tree satisfies property $W$. For the converse it is sufficient, in view of Theorem 1 , to show that if $X$ is a continuum each of whose nondegenerate subcontinua satisfies property $W$, and if $K$ and $L$ are nondegenerate subcontinua of $X$ with $K \subset L$, then $K$ contains a cutpoint of $L$. Now $K$ must have a cutpoint $p$ and so $p$ is not an endpoint of $K$. It follows that $p$ is not an endpoint of $L$ so that, by property $W, p$ is a cutpoint of $L$.

If $X$ is a continuum, $Y$ is a subcontinuum and $p \in Y$, let $\theta(p, Y)$ denote the order of $p$ in $Y$, i.e., $\theta(p, Y)$ is the least cardinal number $n$ such that if $U$ is a neighborhood of $p$ then there exists a neighborhood $V$ of $p$ with $\bar{V} \subset U$ and so that $|\partial V \cap Y| \leq n$. The component number of $p$ in $Y$, denoted $\alpha(p, Y)$, is the number of components of $Y-\{p\}$. It is a theorem of Menger [2] that a metrizable continuum $X$ is a dendrite if and only if $\alpha(p, X)=\theta(p, X)$ for each $p \in X$ for which either of these numbers is finite. Again, this result fails for Hausdorff continua.

THEOREM 3. If $X$ is a continuum then $X$ is a tree if and only if for each subcontinuum $Y$ of $X$ it follows that $\theta(p, Y)=\alpha(p, Y)$ for each $p \in Y$ for which either of these numbers is finite.

ProOF. It is known [4] that each tree satisfies the condition. For the converse it suffices to show that the condition implies property $W$. If $p$ is a noncutpoint of $Y$ then $\alpha(p, Y)=1$; hence $\theta(p, Y)=1$, i.e., $p$ is an endpoint of $Y$.

\section{REFERENCES}

1. H. Kok, Connected orderable spaces, Math. Centre Tracts, no. 49, Amsterdam, 1973.

2. K. Menger, Kurventheorie, 2nd ed., Chelsea, 1967.

3. R. L. Moore, Concerning the cutpoints of continuous curves and other closed and connected point-sets, Proc. Nat. Acad. Sci. U.S.A. 9 (1923), 101-106. 
4. L. E. Ward, Jr., Recent developments in dendritic spaces and related topics, Studies in Topology, Academic Press, San Francisco, 1975, pp. 601-647.

5. G. T. Whyburn, Analytic topology, Amer. Math. Soc. Colloq. Publ., vol. 28, Amer. Math. Soc., Providence, R. I., 1942.

6. R. L. Wilder, Concerning continuous curves, Fund. Math. 7 (1925), 340-377.

Department of Mathematics, University of Oregon, Eugene, Oregon 97403 